\title{
AVALIAÇÃO DA ATIVIDADE ANTIOXIDANTE E ANTIGLICANTE DO EXTRATO DE Tamarindus indica L. E DESENVOLVIMENTO DE FORMULAĈ̃O COSMÉTICA PARA O COMBATE À ACELERAÇÃO DO ENVELHECIMENTO CUTÂNEO
}

\author{
JULIA RICCI, \\ JULIA MARIA CARDOSO BATISTA, \\ FERNANDA BORGES DE ALMEIDA, \\ VERA ISAAC.
}

\begin{abstract}
RESUMO - O processo de envelhecimento da pele tem, como causa, além dos danos causados pelos raios UV, a mudança quantitativa de proteínas da matriz extracelular, que são causadas pela formação de Produtos Finais de Glicação Avançada (AGEs), acumulados ao longo da vida, podendo afetar especialmente proteínas de longa duração, como o colágeno. Como a formação de AGEs e radicais livres estão dependentes das reações de oxidação, a aplicação de antioxidantes e antiglicantes nos produtos cosméticos é uma das estratégias utilizadas para prevenir o envelhecimento, sendo os extratos vegetais, já descritos em literatura, potenciais agentes antioxidantes e antiglicantes. $\mathrm{O}$ objetivo deste trabalho foi investigar o extrato de Tamarindus indica $L$. obtido por diferentes líquidos extratores quanto a sua atividade antiglicante e possível ação contra o envelhecimento cutâneo. Os extratos vegetais foram preparados com a polpa do fruto na concentração de $30 \%$, nos quais foi testada a capacidade extratora de 3 tipos de solventes, sendo eles etanol $70 \%$, água destilada em temperatura ambiente e água destilada a $70^{\circ} \mathrm{C}$, sendo macerados por 24 horas e filtrados. O hidroalcoólico foi rotaevaporado e após, todos liofilizados. Com os extratos liofilizados, foram realizados os ensaios de doseamento de fenólicos totais, utilizando o reagente Folin-Ciocalteau (1:10) e carbonato de sódio (4\%); doseamento de flavonoides, utilizando solução de cloreto de alumínio (2\%) e determinação da atividade antioxidante, com o radical DPPH $\bullet(0,004 \% \mathrm{~m} / \mathrm{v})$. Após, com o melhor solvente extrator para a atividade antioxidante, foi realizado o teste de fotoproteção, a qual foi avaliada in vitro para o extrato já incorporado em formulação cosmética, através de medida do Fator de Proteção Solar (FPS) da formulação utilizando o equipamento Optometrics SPF-290S e a atividade antiglicante, pela determinação de fluorescência de meios de incubação preparados com Albumina de soro Bovino (BSA), tampão fosfato de sódio, azida de sódio, frutose e glicose. Os resultados obtidos mostraram que o extrato hidroalcoólico de $T$. indica devido ao seu conteúdo de biomoléculas, demonstrou atividade antioxidante mesmo que não tão acentuada. Porém, não sendo o bastante para causar efeito fotoprotetor e antiglicação em fitocosméticos.
\end{abstract}

PALAVRAS-CHAVE - Tamarindus indica L.; Tamarindo; Antiglicante; Antioxidante.

\section{INTRODUÇÃO}

O envelhecimento da pele é provocado por fatores intrínsecos e extrínsecos. Cerca de $80 \%$ dos sinais visíveis são provocados pelos raios $\mathrm{UV}$, que quando atingem uma pele desprotegida, podem gerar alterações químicas em moléculas através dos radicais livres, proporcionando a aceleração do envelhecimento cutâneo e podem provocar, também, desenvolvimento de câncer de pele [1].

Por apresentarem valor nutricional alto, frutos e polpas têm sido bastante agregados em pesquisas. A presença de substâncias como os carotenóides, vitamina C e substâncias fenólicas contribuem para manter o equilíbrio entre a produção e eliminação de espécies reativas de oxigênio, o que causa a redução e inibição de lesões causadas por radicais livres nas células e a formação de AGEs [2].

Dentre as mais diversas classes de substâncias antioxidantes de ocorrência natural, os compostos fenólicos têm ganhado grande importância nos últimos anos, uma vez que inibem a peroxidação lipídica e a lipooxigenase in vitro. Sua atividade antioxidante deve-se principalmente às suas propriedades redutoras e estrutura química, desempenhando um papel importante na neutralização ou sequestro de radicais 
livres e quelação de metais de transição, agindo tanto na etapa de iniciação como na propagação do processo oxidativo. Sua ação também gera intermediária, os quais são estáveis por possuírem ressonância do anel aromático em sua estrutura [3]. Na espécie vegetal, os compostos fenólicos enquadramse em diversas categorias, como fenóis simples, ácidos fenólicos (derivados de ácidos benzóico e cinâmico), cumarinas, flavonoides, estilbenos, taninos condensados e hidrolisáveis, lignanas e ligninas [4].

Os flavonoides possuem estrutura ideal para o sequestro de radicais livres, sendo antioxidantes bastante efetivos, com atividade maior que a vitamina $\mathrm{C}$ e $\mathrm{E}$. Sua atividade se deve a fatores como a reação da estrutura como agente doador de hidrogênio e elétrons, a estabilidade do radical flavanoil formado, capacidade de quelar metais de transição, reatividade maior frente a outros antioxidantes, solubilidade e interação com membranas. Quanto mais hidroxilas a estrutura do flavonoide apresentar, maior sua atividade como agente doador de hidrogênio [5].

Acredita-se que o estresse oxidativo induz a formação de AGEs, assim como proteínas glicadas também podem causar estresse oxidativo [6]. Sendo assim, tem-se que elementos que exercem atividade antioxidante também podem exibir atividade antiglicante.

\section{REVISÃO DA LITERATURA}

Mudanças celulares como as alterações quantitativas, oxidação lipídica e glicosilação das proteínas da matriz extracelular são fatores intrínsecos que estão também envolvidos no envelhecimento da pele [7]. Essas mudanças resultam na perda da capacidade de retração e do poder tensor, causando a formação de rugas, aumento da fragilidade e diminuição da cicatrização de feridas [8].

A glicosilação é oriunda da reação de Maillard, envolvendo a redução dos açúcares e o grupo amina dos aminoácidos ou proteínas, formando os produtos de Amadori, os quais representam a glicação inicial. Quando estes produtos passam por reações como reorganização, desidratação e condensação formam derivados fluorescentes irreversíveis, conhecidos como Produtos Finais de Glicação Avançada AGEs, afetando fibras colágenas e elásticas [9].

Uma vez que a formação de AGEs e de radicais livres são dependentes das reações de oxidação, o uso de antioxidantes tais como a vitamina $\mathrm{C}$ e $\mathrm{E}$, e a incorporação de ativos antioxidantes nos produtos cosméticos é uma das estratégias escolhidas para preveni-los, podendo assim prevenir o envelhecimento cutâneo [10].

Pertencente à família das leguminosas e natural de regiões tropicais e subtropicais, o tamarindo (T. indica L.) tem seu uso diversificado pelos mais diferentes tipos de populações. Do tamarindo, são usados desde sua polpa até suas folhas, sendo que há relatos de que algumas populações rurais utilizam sua polpa seca juntamente com as sementes para previnir a peroxidação lipídica, a qual causa a rancidez de óleos como os de amendoim e de coco, assim, aumentando sua vida útil. Em um estudo realizado com moradores do
Município de Sobral - CE foi revelado que a fruta também é utilizada em condições de inflamação, infecção urinária e prisão de ventre [11]. Quimicamente, presume-se que o tamarindo tenha ácido tartárico, carboidratos, pectinas, pirazinas e tiazóis [12], além da presença de compostos responsáveis pela capacidade antioxidante, como 2-hidroxi-3', 4'-di-hidroxiacetofenona, 3,4-di-hidroxibenzoato de metila, acetato de 3,4-di-hidroxifenil e epicatequina [13].

\section{METODOLOGIA}

\section{A. OBTENÇÃO DO EXTRATO VEGETAL}

Os extratos vegetais foram preparados com a polpa do fruto na concentração de $30 \%(\mathrm{~m} / \mathrm{m})$ tendo como solvente etanol $70^{\circ} \mathrm{GL}(\mathrm{E} 70)$, água destilada a $70^{\circ} \mathrm{C}(\mathrm{EAQ})$ e água destilada à temperatura ambiente (EAA). Após, foram submetidos à maceração por 24 horas, à filtração; o hidroetanólico, à rotaevaporação e após, todos foram liofilizados.

\section{B. DOSEAMENTO DE FENÓLICOS TOTAIS}

Utilizou-se o método espectrofotométrico de FolinCiocalteau, onde o ácido gálico era o padrão de referência. Uma alíquota de $0,5 \mathrm{~mL}$ de amostra liofilizada diluída em água, numa concentração de $33 \mathrm{mg} / \mathrm{mL}$, foi transferida para um tubo de ensaio e lhes foram adicionados $2,5 \mathrm{~mL}$ do reagente Folin-Ciocalteau, numa proporção 1:10, com água. Esses reagentes ficaram em repouso de 3 a 8 minutos e a eles foram adicionados $2 \mathrm{~mL}$ de carbonato de sódio a $4 \%$. Após, permaneceram em repouso por um período de 2 horas ao abrigo da luz e a leitura espectrofotométrica foi realizada a $740 \mathrm{~nm}$. Um reagente branco foi feito nas mesmas condições. Realizou-se, também, uma curva analítica contendo 0,1 , 0,08, 0,06, 0,04, 0,02, 0,01 mg. $\mathrm{mL}^{-1}$ de ácido gálico e os resultados expressos em $\mathrm{mg}$ equivalentes de ácido gálico por $100 \mathrm{mg}$ de extrato (EAG.100 mg ${ }^{-1}$ ) [14], [15]. Para cada extrato, o ensaio foi realizado em triplicata.

\section{DOSEAMENTO DE FLAVONOIDES TOTAIS}

Para o doseamento de flavonoides totais, seguiu-se a metodologia descrita por Woisky e Salatino (1998) [16], em que $1 \mathrm{~mL}$ de solução de amostra $33 \mathrm{mg} / \mathrm{mL}$ foi adicionado a $0,5 \mathrm{~mL}$ de solução de cloreto de alumínio $(\mathrm{AlCl} 3)$ a $2 \%$. Esperou-se 15 minutos e as absorbâncias foram lidas a 420 $\mathrm{nm}$. Um reagente branco foi realizado nas mesmas condições. Utilizou-se para o doseamento uma curva analítica de rutina nas concentrações $2 \times 10^{-5}, 3 \times 10^{-5}, 4 \times 10^{-5}, 6 \times 10^{-5}$, $8 \times 10^{-5}$, e $1 \times 10^{-4} \mathrm{mg} \cdot \mathrm{mL}^{-1}$ e quercetina nas concentrações $4 \times 10^{-6}, 8 \times 10^{-6}, 1,2 \times 10^{-5}, 1,6 \times 10^{-5}, 2 \times 10^{-5}$ e $2,4 \times 10^{-5}$ $\mathrm{mg} \cdot \mathrm{mL}^{-1}$. Os resultados foram expressos em equivalentes de quercetina por $100 \mathrm{mg}$ de extrato (EQ.100 $\mathrm{mg}^{-1}$ ) e em equivalentes de rutina por $100 \mathrm{mg}$ de extrato (ER.100 $\mathrm{mg}^{-1}$ ). Para cada extrato, o ensaio foi realizado em triplicata.

\section{DETERMINAÇÃO DA ATIVIDADE ANTIOXIDANTE}

A avaliação da atividade antioxidante foi realizada com o uso do radical 1,1-difenil-2-picrilhidrazila (DPPH•), segundo metodologia descrita por Mensor et al. (2001) [17] e Falcão 
et al. (2006) [18], com modificações. Um mililitro da solução aquosa dos extratos, em diferentes concentrações ( 0 a 33 $\mathrm{mg} / \mathrm{mL}$ ) foi adicionado a $2,5 \mathrm{~mL}$ de solução metanólica de $\mathrm{DPPH}^{*}(0,004 \%)$. As soluções foram mantidas ao abrigo da luz e, após 30 minutos, foi determinada a absorbância das soluções descritas anteriormente, a $515 \mathrm{~nm}$. Uma solução controle foi realizada nas mesmas condições, quando a amostra foi substituída por $1 \mathrm{~mL}$ de água. Como os extratos vegetais em sua maioria apresentam substâncias que conferem coloração, não é descartado que possa haver interferências de absorção no comprimento de onda em que ocorre a reação do radical DPPH•, sendo assim também foi preparado um branco de cor, no qual a solução de $\mathrm{DPPH}^{*}$ foi substituída por 2,5 $\mathrm{mL}$ de metanol.

$\mathrm{O}$ ensaio também foi realizado com a vitamina $\mathrm{C}$ (ácido ascórbico), como padrão antioxidante, em concentrações de 0 a $0,0045 \mathrm{mg} / \mathrm{mL}$. Os ensaios foram realizados em triplicata. A média das absorbâncias destas amostras, descontando seus respectivos brancos de cor, foi usada para o cálculo da porcentagem de inibição do radical DPPH• (\% inibição) [19], sendo:

$$
\text { \% inibição DPPH }{ }^{\bullet}=\frac{\left(\mathrm{A}_{\text {máx }}-\mathrm{A}_{\text {teste }}\right)}{\mathrm{A}_{\text {máx }}} \times 100
$$

Onde, $\mathrm{A}_{m x}$ é a absorbância do DPPH• em $515 \mathrm{~nm}$ na ausência de amostra (controle). A teste é a absorbância do $\mathrm{DPPH} \bullet$ em $515 \mathrm{~nm}$ na presença de amostra.

Com os resultados obtidos pelos cálculos da porcentagem de inibição do radical DPPH•, foram determinados os valores de $\mathrm{IC}_{50}$.

\section{E. DETERMINAÇÃO DA ATIVIDADE ANTIGLICANTE}

Para mensuração da atividade antiglicante, foram preparados meios de incubação contendo Albumina de soro bovino (BSA, $10 \mathrm{mg} / \mathrm{ml}$ ), 0,02\% (p / v) de azida de sódio, glicose (25 mM), frutose $(25 \mathrm{mM})$ com o composto teste, extrato liofilizado de $T$. indica $L$. em solução tampão de fosfato de sódio monobásico monoidratado $(50 \mathrm{mM}, \mathrm{pH} 7,4)$. Foram analisadas 5 concentrações do extrato vegetal $(3,20 ; 6,40$; $9,60 ; 12,80$ e $16 \mathrm{mg} / \mathrm{mL})$ para a determinação do $\mathrm{IC}_{50}$, utilizando como base as concentrações utilizadas na atividade antioxidante. Um controle negativo contendo Albumina de soro bovino (BSA, $10 \mathrm{mg} / \mathrm{ml})$, glicose $(25 \mathrm{mM})$, frutose (25 mM) e veículo ( $50 \mathrm{mM}$ tampão fosfato) foi também preparado uma vez que o BSA é autofluorescente [20], [21].

As amostras foram incubadas a $37^{\circ} \mathrm{C}$ sob abrigo de luz e agitadas periodicamente por um período de 3 dias. A leitura das amostras foi realizada em espectrofotômetro de fluorescência com um comprimento de onda de excitação de $350 \mathrm{~nm}$ e um comprimento de onda de emissão de $450 \mathrm{~nm}$. As fluorescências resultantes dos ensaios realizados com o extrato e o controle negativo tiveram seus cognatos brancos subtraídos para a correção da fluorescência da linha de base.
A porcentagem de inibição da formação de AGE foi calculada através da seguinte fórmula [22].

$$
\% \text { inibição }=\frac{\mathrm{F}_{\text {controle negativo }}-\mathrm{F}_{\text {experimental corrigida }}}{\mathrm{F}_{\text {controle negativo }}}
$$

\section{F. PREPARAÇÃO DA EMULSÃO BASE}

Para avaliação da atividade fotoprotetora do extrato de $T$. indica, foi preparada uma emulsão base, na qual seria incorporado posteriormente o extrato vegetal e filtros solares. Para sua preparação, foi utilizada a técnica de inversão de fases por aquecimento, com os componentes descritos na Tabela 1.

Tabela 1. Composição da emulsão base

\begin{tabular}{|l|l|l|}
\hline Materia-prima & $\begin{array}{l}\text { Formulação } \\
(\boldsymbol{\%} \mathbf{~ m} / \mathbf{n})\end{array}$ & Função \\
\hline Emulfeel® SPG & 3,00 & Emulsionante \\
\hline Dibutyl Adipate & 1,00 & Emoliente \\
\hline Ethylhexyl Stearate & 1,00 & Emoliente \\
\hline $\begin{array}{l}\text { Mangifera Indica } \\
\text { (Mango) Seed } \\
\text { Butter }\end{array}$ & 2,50 & Emoliente \\
\hline Isopropyl Myristate & 4,00 & Emoliente \\
\hline BHT & 0,05 & Antioxidante \\
\hline $\begin{array}{l}\text { Glycerin } \\
\text { Methylparaben }\end{array}$ & 3,00 & Umectante \\
\hline Dissodium EDTA & 0,18 & Conservante \\
\hline $\begin{array}{l}\text { Polyacrylamide } \\
\text { (and) C13-14 }\end{array}$ & 3,00 & Quelante \\
$\begin{array}{l}\text { Isoparaffin (and) } \\
\text { Laureth-7 }\end{array}$ & & Emulsionante \\
\hline Aqua q.s.p. & 100,00 & Veículo \\
\hline
\end{tabular}

As fases aquosa e oleosa foram aquecidas em banhomaria até uma temperatura de aproximadamente $80^{\circ} \mathrm{C}$ e a fase aquosa foi vertida sobre a fase oleosa, com agitação constante.

Para o preparo das emulsões contendo as substâncias com características fotoprotetoras, os filtros solares descritos abaixo foram adicionados na fase oleosa e aquecidos em banho-maria:

- Octyl Metoxicinamato: $8 \%$

- Bis-Ethylhexyloxyphenol Methoxyphenyl Triazine: $2 \%$

As porcentagens definidas de filtros solares foram baseadas no site FPS BASF - Sunscreen Simulator, com o objetivo de obter-se uma emulsão de FPS 20.

No intuito de adicionar o extrato de $T$. indica à emulsão base, o extrato liofilizado da espécie vegetal foi solubilizado em uma pequena quantidade de água subtraída da quantidade total necessária para a formulação e adicionado na emulsão quando já finalizada e resfriada. 
Dessa forma, foram preparadas as seguintes emulsões:

1) Emulsão base

2) Emulsão Base com filtros solares (Octyl Metoxicinamato e Bis-Ethylhexyloxyphenol Methoxyphenyl Triazine)

3) Emulsão base com extrato de T. indica $2 \%$

4) Emulsão base com extrato T. indica $0,2 \%$

5) Emulsão base com filtros solares e extrato T. indica $2 \%$

6) Emulsão base com filtros solares e extrato T. indica $0,2 \%$

As concentrações do extrato adicionadas às emulsões base foram feitas conforme rotina do grupo.

\section{G. AVALIAÇÃO DA ATIVIDADE FOTOPROTETORA}

A atividade fotoprotetora foi avaliada através da medição do fator de proteção solar (FPS) das formulações utilizando o equipamento Optometrics SPF-290S. Foram aplicados 110 $\mathrm{mg}$ das emulsões previamente preparadas sobre uma fita Transpore ${ }^{\circledR}$ e espalhadas com o objetivo de se obter um filme uniforme de $2 \mathrm{mg} / \mathrm{cm}^{2}$. Em seguida, as amostras foram submetidas à análise pelo equipamento, onde há incidência de radiação ultravioleta por uma lâmpada de Xenônio em uma faixa de comprimento de onda entre 290 a $400 \mathrm{~nm}$. Para cada amostra, as leituras foram realizadas em triplicata e o fator de proteção solar foi calculado com o software SPF [23], [24].

\section{H. ANÁLISE ESTATÍSTICA}

A análise dos dados foi realizada pela determinação de média e desvio padrão, além de análise de variância ANOVA seguida pelo teste de Tukey $(\mathrm{p}<0,05)$, quando adequado.

\section{RESULTADOS}

\section{A. EXTRAÇÃO DO MATERIAL VEGETAL}

Os solventes escolhidos para a extração foram baseados na sua afinidade molecular com as biomoléculas que apresentam atividade antioxidante, assim como também pela posterior possibilidade de incorporação a emulsões, sem que afetasse a saúde do usuário do produto. Os solventes utilizados também são caracterizados como baixo custo e alta disponibilidade [25], [26].

A liofilização do extrato vegetal se fez necessária para promover uma maior estabilidade da fruta, impedindo processos enzimáticos que possam diminuir os princípios ativos de interesse. Além disso, o processo auxilia na concentração de princípios ativos, os quais ficarão em uma menor quantidade de massa da matéria prima [27]. Como resultado, após liofilização obteve-se um produto de mesmo aspecto para os três líquidos extratores, uma massa marrom, de aspecto elástico.

\section{B. DOSEAMENTO DE FENÓLICOS TOTAIS}

Para os cálculos das concentrações de compostos fenólicos totais contidos no extrato foi utilizada a curva analítica de ácido gálico (Gráfico 1).

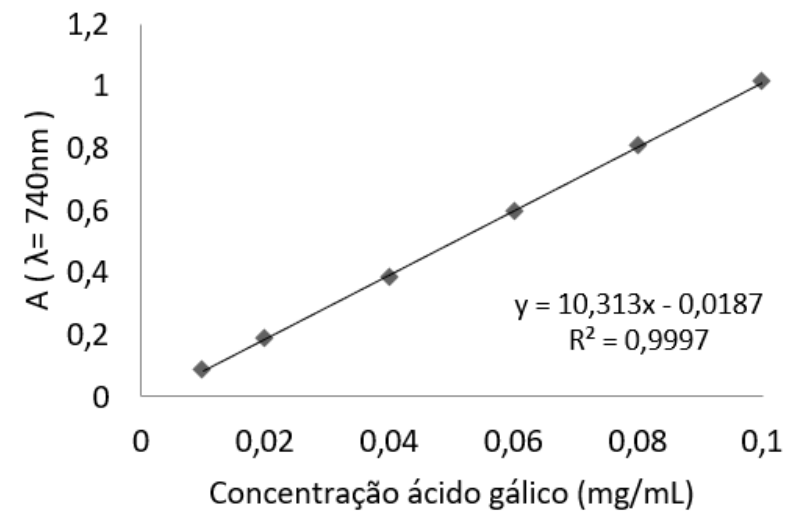

Figura 1. Curva analítica do ácido gálico.

As concentrações de compostos fenólicos contidos nos extratos estão listadas na Tabela 2. Os resultados estão expressos em mg de compostos fenólicos equivalentes ao ácido gálico por $100 \mathrm{mg}$ de extrato.

Tabela 2. Concentração de compostos fenólicos equivalentes ao ácido gálico nos extratos avaliados

\begin{tabular}{|l|l|l|l|l|}
\hline & 1 & 2 & 3 & Média \pm DP \\
\hline EAQ & 7,71 & 7,63 & 7,67 & $7,67^{a} \pm 0,004$ \\
\hline EAA & 7,10 & 7,20 & 7,30 & $7,20^{b} \pm 0,10$ \\
\hline E70 & 9,85 & 9,79 & 9,76 & $9,80^{c} \pm 0,04$ \\
\hline
\end{tabular}

$\mathrm{a}, \mathrm{b}, \mathrm{c}$, sendo $\mathrm{p}<0,01$, a média da concen-

tração de compostos fenólicos equivalentes

ao ácido gálico nos diferentes extratos di-

ferem entre si.

Os resultados demonstraram que o solvente mais efetivo em extrair os compostos fenólicos totais dos extratos liofilizados de T. indica foi o etanol $70^{\circ} \mathrm{GL}$, seguido pela água quente e, finalmente, água à temperatura ambiente, em virtude das diferenças de polaridade e, também, pela temperatura.

Já foi demonstrado que há uma forte influência na extração de flavonoides, uma das substâncias pertencentes à classe dos fenólicos totais, pela concentração de etanol e água, sendo que quando o volume de etanol é menor que $75 \%(\mathrm{v} / \mathrm{v})$, essa extração aumenta [28].

A extração de substâncias bioativas pode ser afetada pela temperatura, sendo que um aumento causa diminuição do conteúdo total de compostos fenólicos [29]. Porém, estudos afirmaram que o aquecimento das sementes de uva favorece a liberação de compostos fenólicos, aumentando sua concentração em seus extratos [30]. Sendo assim, o processo de extração de antioxidantes naturais constitui mecanismo complexo. Portanto é possível inferir que, tanto o etanol quanto a água são solventes capazes de extrair os compostos fenólicos, entretanto, a mistura destes dois solventes indica maior capacidade de extração de compostos fenólicos de $T$. Indica. 


\section{DOSEAMENTO DE FLAVONOIDES TOTAIS}

Para a construção da curva analítica utilizou-se como padrão a rutina e a quercetina. Através da curva analítica da rutina (Gráfico 2) e da quercetina (Gráfico 3), foi possível o cálculo das quantidades de flavonoides presentes nos extratos, ilustrados na Tabela 3 , sendo expressos como mg de flavonoides equivalentes à rutina por $100 \mathrm{mg}$ de extrato e, na Tabela 4, em $\mathrm{mg}$ de flavonoides equivalentes à quercetina por $100 \mathrm{mg}$ de extrato.

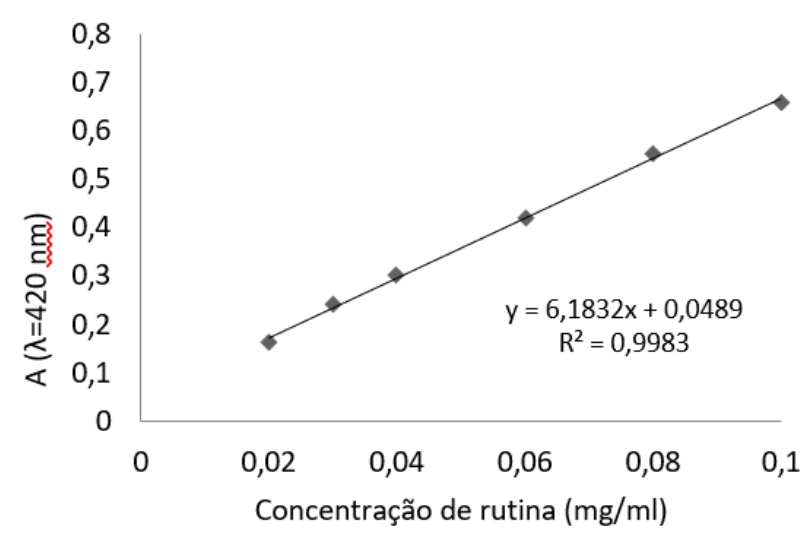

Figura 2. Curva analítica da rutina.

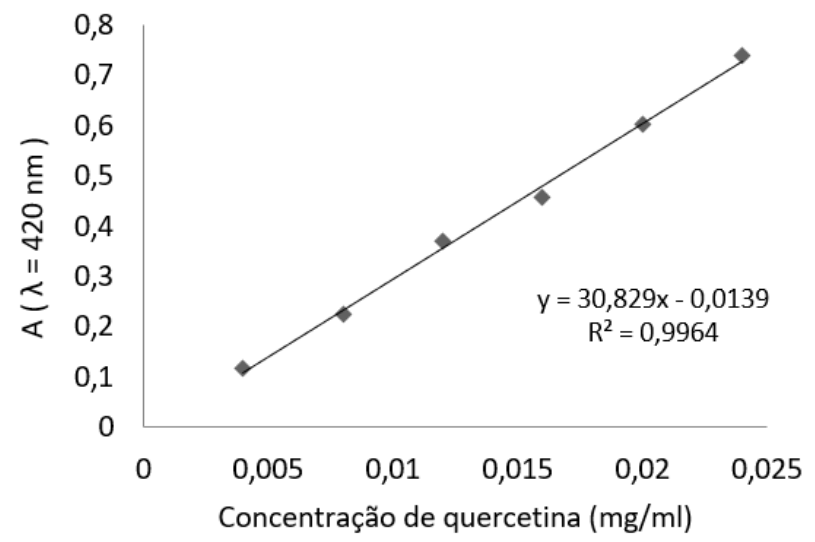

Figura 3. Curva analítica da quercetina.

Tabela 3. Concentração de flavonoides equivalentes à rutina nos extratos avaliados.

\begin{tabular}{|l|l|l|l|l|}
\hline & 1 & 2 & 3 & Média \pm DP \\
\hline EAQ & 2,50 & 2,67 & 2,66 & $2,61^{a} \pm 0,09$ \\
\hline EAA & 1,30 & 1,40 & 1,40 & $1,36^{b} \pm 0,05$ \\
\hline E70 & 8,34 & 8,19 & 8,32 & $8,29^{c} \pm 0,05$ \\
\hline $\begin{array}{l}\text { a, b, c, sendo p <0,01, a média da concen- } \\
\text { tração de flavonoides equivalentes à rutina } \\
\text { nos diferentes extratos diferem entre si. }\end{array}$ \\
\hline
\end{tabular}

Da mesma forma como verificado no doseamento de compostos fenólicos, E70 apresentou uma maior concentração de flavonoides do que os extratos aquosos. Também foi possível
Tabela 4. Concentração de flavonoides equivalentes à quercetina nos extratos.

\begin{tabular}{|l|l|l|l|l|}
\hline & 1 & 2 & 3 & Média \pm DP \\
\hline EAQ & 0,6 & 0,7 & 0,7 & $0,66^{a} \pm 0,05$ \\
\hline EAA & 0,4 & 0,5 & 0,5 & $0,46^{b} \pm 0,05$ \\
\hline E70 & 2,2 & 2,1 & 2,2 & $2,16^{c} \pm 0,05$ \\
\hline
\end{tabular}

$\mathrm{a}, \mathrm{b}, \mathrm{c}$, sendo $\mathrm{p}<0,01$, a média da concentração de flavonoides equivalentes à quercetina nos diferentes extratos diferem entre si.

observar que a concentração de flavonoides equivalentes à rutina presente em todos os extratos foi superior à concentração de flavonoides equivalentes à quercetina.

A quercetina é um dos flavonoides antioxidantes mais potentes devido à quantidade de hidroxilas substituídas em sua molécula. A rutina se difere da quercetina por possuir uma molécula de açúcar (rutinose) em sua posição 3 do anel pirano [31].

Como citado acima, o poder antioxidante do extrato de $T$. indica poderia ser mais acentuado caso o teor de flavonoides equivalentes a quercetina fosse maior.

\section{DETERMINAÇÃO DA ATIVIDADE ANTIOXIDANTE}

No ensaio de atividade antioxidante usando o radical DPPH•, foi utilizado o ácido ascórbico na curva analítica como padrão antioxidante. No Gráfico 4 pode ser visualizado o gráfico da porcentagem de inibição do radical $\mathrm{DPPH} \bullet$ pelo ácido ascórbico.

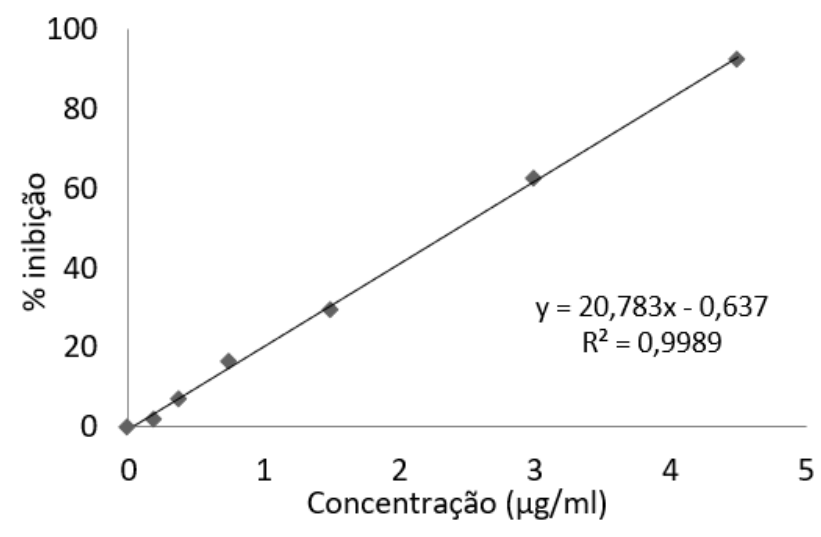

Figura 4. Percentagem de inibição do radical DPPH• por ácido ascórbico, uma substância de referência.

$\mathrm{O} \mathrm{IC}_{50}$ da atividade sequestrante do radical $\mathrm{DPPH} \bullet$ com o uso da vitamina $C$ foi de $2,3755 \times 10^{-3} \mathrm{mg} / \mathrm{mL}$.

Em seguida, foi determinada a atividade antioxidante dos três extratos de T. indica a partir das curvas obtidas no Gráfico 5 , e a equação da reta forneceu informações suficientes para que fosse calculada a quantidade de extrato necessária para que fosse alcançada a inibição de $50 \%$ dos radicais livres 
presentes no meio de análise, gerando os valores de $\mathrm{IC}_{50}(\mathrm{mg}$ de extrato/mL) como pode ser visualizado na Tabela 5.

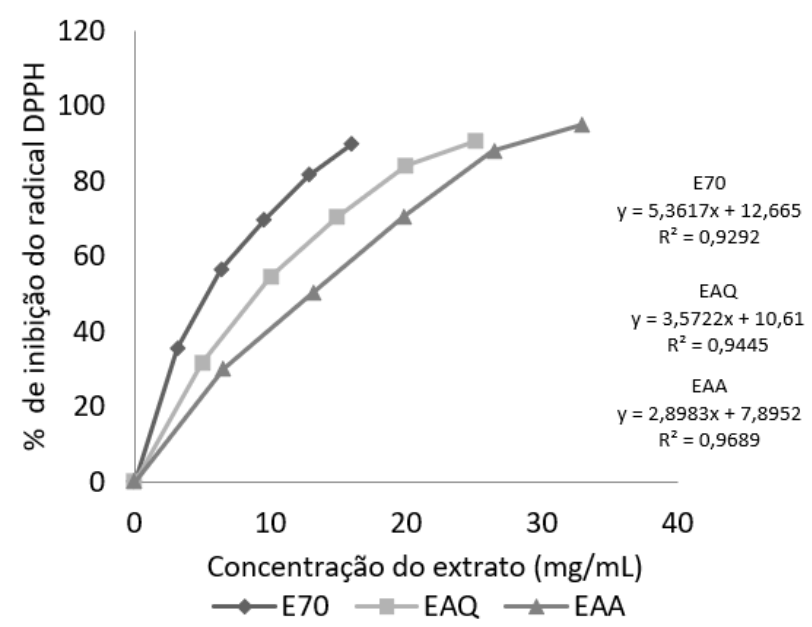

Figura 5. Atividade sequestrante do radical $\mathrm{DPPH} \bullet$ pelos extratos de $T$. indica.

Tabela 5. Valores de IC50 dos extratos avaliados ( $\mathrm{mg}$ de extrato/mL).

\begin{tabular}{|c|c|c|c|c|}
\hline & 1 & 2 & 3 & Média \pm DP \\
\hline$\overline{\mathrm{EAQ}}$ & 11,49 & 9,21 & 9,44 & $10,04^{a} \pm 1,25$ \\
\hline EAA & 14,69 & 14,14 & 14,52 & $14,45^{b} \pm 0,28$ \\
\hline E70 & 6,88 & 6,96 & 7,57 & $7,13^{c} \pm 0,37$ \\
\hline \multicolumn{5}{|c|}{$\begin{array}{l}\mathrm{a}, \mathrm{b}, \mathrm{c} \text {, sendo } \mathrm{p}<0,01 \text {, a média dos valores } \\
\text { de IC50 nos diferentes extratos diferem } \\
\text { entre si. }\end{array}$} \\
\hline
\end{tabular}

Os resultados da atividade antioxidante dos extratos podem ser comparados à concentração dos compostos fenólicos totais e flavonoides totais presentes, já que é observada uma correlação entre a quantidade de compostos fenólicos e flavonoides com a atividade antioxidante. Portanto, ao compararem-se os valores de $\mathrm{IC}_{50}$, E70 possui o menor valor em menor concentração de extrato, seguido do EAQ e EAA. $\mathrm{O} \mathrm{IC}_{50}$ é uma medida de potência de inibição, sendo que o menor valor encontrado dentre os extratos, no caso E70, ainda é maior que o $\mathrm{IC}_{50}$ do ácido ascórbico, porém ainda há atividade antioxidante.

Nos resultados encontrados por Simionatto et al (2015) [32], o extrato da polpa de T. indica $L$. foi inativo frente ao radical livre DPPH•, já o extrato preparado com a casca da fruta revelou um $\mathrm{IC}_{50}$ de $1,9 \mathrm{mg} / \mathrm{mL}$. Ao se comparar ambos os trabalhos, pode-se afirmar que o conteúdo de biomoléculas dos frutos de T. Indica, é variável.

Gobbo-Neto Lopes (2007) [33] afirmaram que os metabólitos secundários nas espécies vegetais são dependentes de fatores como genética, ambiente físico, condições de secagem, temperatura e solo, o que condiz, uma vez que no trabalho citado anteriormente, os frutos foram colhidos em uma região de cerrado, no estado de Mato Grosso do Sul, e os frutos deste presente trabalho foram coletados no interior do estado de São Paulo.

\section{E. DETERMINAÇÃO DA ATIVIDADE ANTIGLICANTE}

A relação dose dependência do extrato de $T$. indica $L$. E70 com a antiglicação, assim como seu $\mathrm{IC}_{50}$ podem ser vistos na Tabela 6 e Gráfico 6.

Tabela 6. Valores de IC50 dos extratos avaliados (mg de extrato/mL).

\begin{tabular}{|l|l|l|l|l|}
\hline & 1 & 2 & 3 & Média \pm DP \\
\hline E70 & 153,99 & 142,94 & 152,47 & $149,80 \pm 5,98$ \\
\hline
\end{tabular}

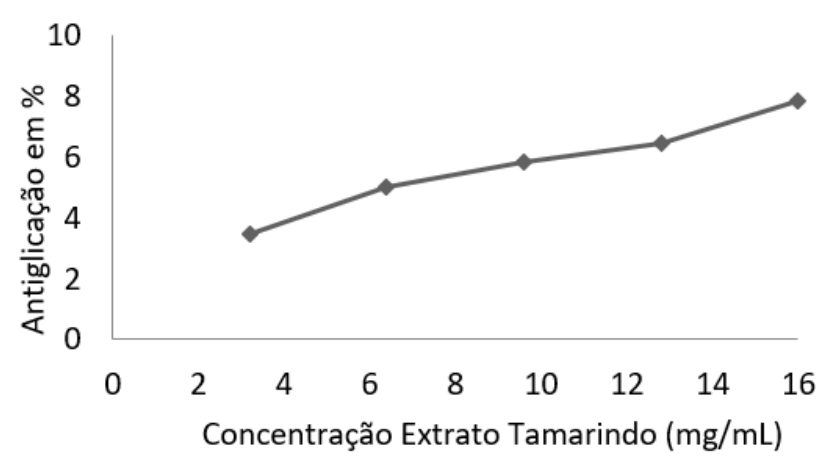

Figura 6. Relação dose dependêncide E70 e a atividade antiglicante.

Os resultados obtidos mostraram que E70 apresentou um $\mathrm{IC}_{50}$ de $149,80 \pm 5,98 \mathrm{mg} / \mathrm{mL}$ para a antiglicação, no entanto, Melo (2015) [34] utilizando um método bastante parecido ao apresentado, demonstrou resultados melhores que os do presente trabalho, obtendo um $\mathrm{IC}_{50}$ de $0,0206 \pm 0,05 \mathrm{mg} / \mathrm{mL}$ para a aminoguanidina, um composto com capacidade antiglicante já conhecida, e $0,0381 \pm 0,93 \mathrm{mg} / \mathrm{mL}$ para o extrato de Mimosa tenuiflora Wild., planta pertencente à família de leguminosas assim como o $T$. indica.

Tem-se então que mesmo o melhor líquido extrator para a atividade antioxidante do extrato de $T$. indica não apresentou atividade antiglicante, uma vez que seu valor de $\mathrm{IC}_{50}$ foi muito mais alto que compostos com capacidade antiglicante já conhecida.

De acordo com Srichairatanakool (2009) [35], é possível que o efeito antiglicante de extratos vegetais seja causado por substâncias como a rutina, galato, pirogalato e catequina, por competirem com a glicose para se ligar às moléculas de proteína, levando a inibição da glicação proteíca. É proposto também que exemplos de estratégias antiglicação sejam o uso de sequestradores de alfa-oxoaldeído e indutores de defesa enzimática [36].

\section{F. AVALIAÇÃO DA ATIVIDADE FOTOPROTETORA}

A presença de sustâncias ativas como as de poder antioxidante, incluindo vitaminas $\mathrm{C}$ e $\mathrm{E}$, os taninos, alcaloides e flavonoides, fazem com que os vegetais possam ser empregados em formulações cosméticas a fim de proporcionar fotoproteção cutânea [37]-[39]. Portanto, escolheu-se E70 
para prosseguir com o experimento, já que apresentou o menor IC50 na capacidade antioxidante quando comparado à EAQ e EAA.

Com o preparo das emulsões, as que possuíam o extrato de tamarindo, apresentaram aspecto levemente alaranjado, já as com filtro solar, possuíam aparência acentuada amarela. A emulsão base sem nenhuma adição possuiu aspecto comum, esbranquiçado.

Os resultados da investigação da atividade fotoprotetora estão demostrados na Tabela 7, onde são apresentados os FPS Optometrics SFP 290 土 Desvio Padrão das formulações avaliadas, assim como o FPS teórico e real Sunscreen Simulator - BASF da emulsão base preparada com os filtros solares prédeterminados.

Tabela 7. FPS Optometrics SFP $290 \pm$ DP das formulações avaliadas e FPS teórico e real Sunscreen Simulator - BASF.

\begin{tabular}{|l|l|l|}
\hline Formulações & $\begin{array}{l}\text { FPS BASF } \\
\text { Sunscreen } \\
\text { Simulator }\end{array}$ & FPS Optometrics \\
\hline $\begin{array}{l}\text { Emulsão base } \\
\text { sem filtros }\end{array}$ & - & $1,03 \pm 0,01$ \\
\hline $\begin{array}{l}\text { Emulsão base + } \\
\text { filtros }\end{array}$ & 20 & $23,38 \pm 0,64$ \\
\hline $\begin{array}{l}\text { Emulsão base }+ \\
\text { extrato 0,2\% }\end{array}$ & - & $1,03 \pm 0,01$ \\
\hline $\begin{array}{l}\text { Emulsão base + } \\
\text { extrato 2\% }\end{array}$ & - & $1,09 \pm 0,02$ \\
\hline $\begin{array}{l}\text { Emulsão base + } \\
\text { filtros + extrato } \\
0,2 \%\end{array}$ & - & $21,08 \pm 1,69$ \\
\hline $\begin{array}{l}\text { Emulsão base }+ \\
\text { filtros + extrato } \\
\text { 2\% }\end{array}$ & - & $24,88 \pm 0,74$ \\
\hline $\begin{array}{l}\text { Sendo p >0,05, a média de FPS das emul- } \\
\text { sões que continham apenas os filtros solares } \\
\text { e as que continham filtros solares }+ \text { extrato } \\
\text { de T. indica, não diferem entre si. }\end{array}$ \\
\hline
\end{tabular}

Com base nos resultados obtidos é possível inferir que o extrato de $T$. indica, mesmo em maior concentração (2\%), não apresentou aumento significativo no FPS das emulsões avaliadas. Também é possível observar que não houve sinergismo quando o extrato da fruta foi incorporado a uma emulsão com filtros solares.

A investigação da fotoproteção de compostos naturais se deve, principalmente, à capacidade de absorção pelos cromóforos de algumas de suas moléculas da radiação ultravioleta, associadas à atividade antioxidante [40]. Carotenoides e flavonoides são exemplos de constituintes de frutas que contribuem para a prevenção contra os efeitos deletérios da radiação ultravioleta [41], [42].

No presente trabalho, mesmo apresentando atividade antioxidante, o extrato de $T$. indica não pode ser considerado como um fotoprotetor. Segundo Violante et al. (2009) [39], o baixo FPS de extratos vegetais é explicado devido a sua constituição complexa de moléculas ativas e menos ativas, podendo apresentar pouca concentração de moléculas com capacidade de absorver a radiação UV assim como destacando também a dificuldade da determinação da absorção máxima dos extratos vegetais.

\section{CONCLUSÕES}

Com os resultados obtidos no presente trabalho é possível inferir que dentre os 3 líquidos extratores testados, o etanol $70^{\circ} \mathrm{GL}$ foi o melhor solvente para extração das biomoléculas responsáveis pela atividade antioxidante do extrato de $\mathrm{T}$. indica, que mesmo não acentuada, ainda é presente.

$\mathrm{O}$ resultado negativo também para a atividade antiglicante e fotoprotetora demonstra que o extrato da polpa de T. indica, preparado na concentração de $30 \%(\mathrm{~m} / \mathrm{m})$, através de maceração, não possuiu potencial ação contra o envelhecimento cutâneo; porém, os resultados poderiam ser diferentes devido a variáveis como época e local da colheita da fruta, parte da planta utilizada, concentração do extrato e método de extração.

\section{Referências}

[1] A. F. Bem; J. Kunde. A importância da determinação da hemoglobina glicada no monitoramento das complicações crônicas do diabetes mellitus. Jornal Brasileiro de Patologia e Medicina Laboratorial, 42(3):185-191, 2006.

[2] G. A. Maia; P. H. M. Sousa; A. S. Lima. Processamento de sucos de frutas tropicais. Fortaleza: Editora UFC, 2007.

[3] S. E. Soares. Ácidos fenólicos como antioxidantes. Revista de Nutrição, 15(1):71-81,2002.

[4] E. Haslam. Natural Polyphenols (Vegetable Tannins) as Drugs: Possible Modes of Action. Journal of Natural Products, 59(2):205, 1996.

[5] A. I. B. S. Barreiros; J. P. David; J. M. David. Estresse oxidativo: Relação entre geração de espécies reativas e a defesa do organismo. Química nova, 29:113-123, 2006.

[6] J. W. Baynes; S. R. Thorpe. Role of oxidative stress in diabetic complications: a new perspective on an old paradigma. Diabetes, 48(1):1-9, 1999.

[7] J. Wautier; P. Guillausseau. Advanced glycation end products, their receptors and diabetic angiopathy. Diabetes Metabolism, 535-542, 2010.

[8] G. Jenkins. Molecular mechanisms of skin ageing. Mechanisms of Ageing and Development,123(7):801-810, 2002.

[9] S. Yamagishi; T. Matsui; H. Adachi; M. Takeuchi. Positive association of circulating levels of advanced glycation end products (AGE) with pigment epithelium-derived fator (PEDF) in a general population. Pharmacological Research, 6(2):103-107, 2010.

[10] A. R. G. Parrinha. Novas tendências em Cosmética Anti-Envelhecimento Dissertação (Mestrado Integrado em Ciências Farmacêuticas) - Escola de Ciências e Tecnologia da Saúde, Universidade Lusófona de Humanidades e Tecnologias, Lisboa, 2014.

[11] A. H. Teixeira; M. M. Bezerra; H. V. Chaves; D. R. Val; S. M. P. Filho; A. A. R. Silva. Conhecimento popular sobre o uso de plantas medicinais no município de Sobral -Ceará, Brasil. Sanare Revista de Políticas Públicas, 13(1):23-28, 2014.

[12] W. Abebe. Herbal medication: potential for adverse interactions with analgesic drugs. Journal of Clinical Pharmacy Therapeutics, 27:391-401, 2002 .

[13] T. Tsuda; Y. Fukaya; K. Ohshima; A. Yamamoto; S. Kawakishi; T Osawatsuda. Antioxidative activity of tamarind extract prepared from the seed coat (Japanese). Journal of the Japanese Society for Food Science and Technology, 42:430-435, 1995.

[14] G. Kroyer; N. Hegedus. Evaluation of bioactive properties of pollen extracts as functional dietary food supplement. Innovative Food Science Emerging Technologies, 2(3):171-174, 2001. 
[15] V. L. Singleton; A. Joseph; J. Rossi. Colorimetry of total phenolics with phosphomolibdic - phosphotungstic acid reagents. American Journal of Enology and Viticulture,16:144-149, 1965.

[16] R. G. Woisky; A. Salatino. Analysis os propolis: some parameters ond prodecore for chemical fuality control. Journal of apicultural research bee world, 37(2):99-105, 1998.

[17] L. L. Mensor; F. S. Menezes; G. G. Leitão; A. S. Reis; T. C. Santos; C. S. Coube; S. G. Leitão. Screening of brazilian plant extracts for antioxidant: Activity by the use of DPPH free radical method. Phytotherapy. Research, 15:127-130, 2001.

[18] D. Q. Falcão; E. R. Costa; D. S. Alviano; C. S. Alviano; R. M. Kuster; F. S. Menezes. Atividade antioxidante e antimicrobiana de Calceolaria chelidonioides Humb. Bonpl. Kunth. Revista Brasileira de Farmacognosia, 16:73-76, 2006.

[19] P. Molyneux. The use of the stable free radical diphenylpicrylhydrazyl (DPPH) for estimating antioxidant activity. Songklanakarin Journal of Science and Technology, 26(2):211-219, 2004.

[20] C. H. Wu; G. C. Yen. Inhibitory Effect of Naturally Occurring Flavonoids on the Formation of Advanced Glycation Endproducts. Journal of Agricultural and Food Chemistry, 53(8):3167-3173, 2005.

[21] J. A. Vinson; T. B. Howard. Inhibition of protein glycation and advanced glycation end products by ascorbic acid and other vitamins and nutrients. Nutricional Biochemistry, 7:659-663, 1996.

[22] L. P. Beaulieu; C. S. Harris; A. Saleem; A. Cuerrier; P. S. Haddad; L. C. Martineau; S. A. L. Bennett; John T. Arnason. Inhibitory effect of the cree tradicional medicine wiishichimanaanh (Vaccinium vitis-idaea) on advanced glycation endproduct formation: identification of active principles. Phytotherapy Research, 24(5):741-747, 2010.

[23] S. Kale; P. Ghoge; A. Ansari; A. Waje; A. Sonawane. Formulation and in-vitro determination of sun protection factor of Nigella sativa Linn. seed oil sunscreen cream. International Journal of PharmTech Research, 2(4):2194-2197, 2010.

[24] A. Duraisamy; N. Narayanaswamy; A. Sebastian; K. Bakakrishnan. Sun Protection and Anti- Inflammatory Activities of some Medicinal Plants. International Journal of Pharmaceutical Review and Research, 1(1):13-16, 2011.

[25] D. Andreo; N. Jorge. Antioxidantes naturais: técnicas de extração. Boletim do Centro de Pesquisa de Processamento de Alimentos, 24(2):319-336, 2006.

[26] L. Wang; C. L. Weller. Recent advances in extraction of nutraceuticals from plants. Trends in Food Science and Technology, 17:300-312, 2006.

[27] B. G. Chiari. Desenvolvimento, avaliação da eficácia e segurança de fitocosmético contendo extrato de Psidiumguajava L. Dissertação de Mestrado. Faculdade de Ciências Farmacêuticas - Unesp. Araraquara/SP, 2011.

[28] Z. Shouqin; X. Jun; W. Changzheng. High hydrostatic pressure extraction of flavonoids from própolis. Journal of Chemical Technology Biotechnology, 80:50-54, 2005.

[29] E. Conde; E. Cadahía; M. C. García-Vallejo; B. F. Simón. Polyphenolic composition of Quercus suber cork from different spanish provenances. Journal of Agricultural and Food Chemistry, 46(8):3166-3171, 1998. b30 S. Y. Kim; S. M. Jeong; W. P. Park; K. C. Nam; D. U. Ahn; S. C. Lee. Effect of heating conditions of grape seeds on the antioxidant activity of grapes seed extracts. Food Chemistry, 97(3):472-479, 2006.

[30] A. J. F. Souza. Avaliação dos efeitos antimicrobianos de Rutina e Quercetina in vitro. Dissertação (mestrado) - Universidade Estadual de Campinas. Instituto de Biologia. Departamento de Bioquímica, Campinas, 2009.

[31] E. Simionatto; S. C. Hess; M. T. L. P. Peres; R. A. Silva. Atividade antioxidante e teor de fenois totais dos frutos de Tamarindus indica. Sociedade Brasileira de Química ( SBQ) 31a Reunião Anual da Sociedade Brasileira de Química. Águas de Lindóia. 2008.

[32] L. Gobbo-Neto; N. P. Lopes. PLANTAS MEDICINAIS: FATORES DE INFLUÊNCIA NO CONTEÚDO DE METABÓLITOS SECUNDÁRIOS. Quimica Nova, 30(2):374-381, 2007.

[33] I. S. V. Melo. Determinação da atividade antiglicante de extratos de plantas sobre produtos de glicação avançada (AGE) in vitro. Tese (Doutorado em Rede Nordeste de Biotecnologia) - Instituto de Química e Biotecnologia, Programa de Pós Graduação em Rede Nordeste de Biotecnologia, Universidade Federal de Alagoas, Maceió, 2015.

[34] S. Srichairatanakool. Antioxidative Activity, Polyphenolic Content and Anti-Glycation Effect of Some Thai Medicinal Plants Traditionally Used in Diabetic Patients. Medicinal chemistry, 5:139-47, 2009.
[35] P. J. Thornalley. Glycation in diabetic neuropathy: characteristics, consequences, causes and therapeutic options. International Review of Neurobiology, 50:37-57, 2002.

[36] C. M. O. Simões; E. P. Schenkel; J. C. P. Mello; L. A. Mentz; P. R. Petrovick. Farmacognosia: do produto natural ao medicamento. 1 ed. Porto Alegre: Editora Artmed, 2017.

[37] M. B. Rosa; T. G. Oliveira; C. A. Carvalho; F. D. Silva; L. M. Carvalho; P. C. Nascimento; R. L. Peres. Estudo espectrofotométrico da atividade fotoprotetora de extratos aquosos de Achillea millefolium, Brassica oleracea var. capitata, Cyperus rotundus, Plectranthus barbatus, Porophyllum ruderale (JACQ) cass e Sonchus Oleraceus. Revista Eletrônica de Farmácia, $1: 101-10,2008$.

[38] I. M. P. Violante; I. M. Souza; C. L. Venturini; A. F. S. Ramalho; R. A. N. Santos; M. Ferrari. Avaliação in vitro da atividade fotoprotetora de extratos vegetais do cerrado de Mato Grosso. Revista Brasileira de Farmacognosia, 19(2A):452-457, 2009.

[39] T. Guaratini; D. R. Callejon; D. C. Pires; J. N. C. Lopes; L. M. Lima; D. G. Neto; C. Sustovich; N. P. Lopes. Fotoprotetores derivados de produtos naturais: perspectivas de mercado e interações entre o setor produtivo e centros de pesquisa. Química Nova, 32(3):717-721, 2009.

[40] W. Stahl; H. Sies. Carotenoids and protection against solar UV radiation. Skin Pharmacology and Physiology, 15(5):291-296, 2002.

[41] K. R. Markhan; K. G. Ryan; S. J. Bloor; K. A. Mitchell. An increase in the luteolin: apigenin ratio in Marchantia polymorpha on UV-B enhancement Phytochemistry, 48:791-794, 1998.

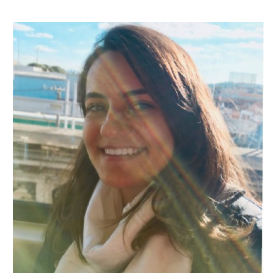

JULIA RICCI

Universidade Estadual Paulista (UNESP), Faculdade de Ciências Farmacêuticas.

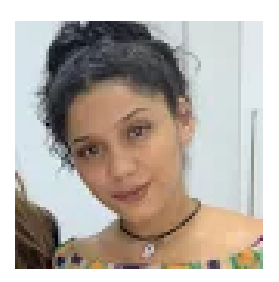

JULIA MARIA CARDOSO BATISTA

Universidade Estadual Paulista (UNESP), Faculdade de Ciências Farmacêuticas.

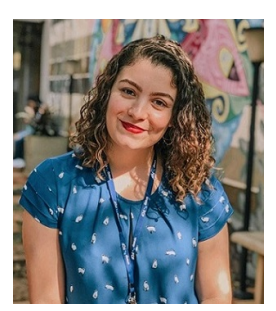

FERNANDA BORGES DE ALMEIDA

Universidade Estadual Paulista (UNESP), Faculdade de Ciências Farmacêuticas. 
VERA ISAAC

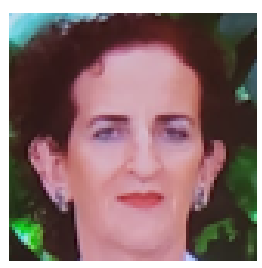

Universidade Estadual Paulista (UNESP), Facul-

dade de Ciências Farmacêuticas. 